\title{
Article \\ Quantitative and Qualitative Image Analysis of In Vitro Co-Culture 3D Tumor Spheroid Model by Employing Image-Processing Techniques
}

\author{
Mukta Sharma 1,*(D), Venkanagouda S. Goudar ${ }^{2}$, Manohar Prasad Koduri ${ }^{3,4} \mathbb{D}$, Fan Gang Tseng 2,5 (D) \\ and Mahua Bhattacharya ${ }^{1}$
}

Citation: Sharma, M.; Goudar, V.S.; Koduri, M.P.; Tseng, F.G.; Bhattacharya, M. Quantitative and Qualitative Image Analysis of In-Vitro Co-Culture 3D Tumor Spheroid Model by Employing ImageProcessing Techniques. Appl. Sci. 2021, 11, 4636. https://doi.org/ 10.3390/app11104636

Academic Editors: Leonardo Rundo, Carmelo Militello and Andrea Tangherloni

Received: 24 February 2021

Accepted: 31 March 2021

Published: 19 May 2021

Publisher's Note: MDPI stays neutral with regard to jurisdictional claims in published maps and institutional affiliations.

Copyright: (C) 2021 by the authors Licensee MDPI, Basel, Switzerland. This article is an open access article distributed under the terms and conditions of the Creative Commons Attribution (CC BY) license (https:// creativecommons.org/licenses/by/ $4.0 /)$.
1 Department of Information Technology, ABV-Indian Institute of Information Technology and Management, Gwalior, Madhya Pradesh 474015, India; mb@iiitm.ac.in

2 Department of Engineering and System Sciences, National Tsing Hua University, Hsinchu 30013, Taiwan; vsg@gapp.nthu.edu.tw (V.S.G.); fangang@ess.nthu.edu.tw (F.G.T.)

3 Department of Mechanical, Materials and Aerospace, School of Engineering, University of Liverpool, Harrison Hughes Building, Liverpool L693GH, UK; mfgtjcjh@liverpool.ac.uk

4 International Intercollegiate PhD Program, National Tsing Hua University, Hsinchu 30013, Taiwan

5 Research Center for Applied Sciences, Academia Sinica, Taipei, Frontier Research Center on Fundamental and Applied Sciences of Matters, National Tsing Hua University, Hsinchu 30013, Taiwan

* Correspondence: mukta@iiitm.ac.in

Abstract: This work proposes a novel region-estimation (RE) algorithm using the quantification of colon-cancer (HCT-8) and fibroblasts (NIH3T3) cells to estimate the densest region of colon-cancer cells in in vitro 3D co-cultured spheroids. Cells were labelled with different cell tracker dyes to track the cells. The technique involves staining cells with cell trackers The quantification of HCT-8 and NIH3T3 cells by the RE algorithm leads to distribution pattern analysis of cells from the core to the periphery, which ultimately estimates the densest region of HCT-8 cells in an in vitro 3D cell spheroid. Cell quantification by the RE algorithm was compared with the results of cell quantification by Image software. Results demonstrated the distribution patterns of cells from the core to the peripheral region of the in vitro 3D cell spheroid. The overall experimentation showed that the proposed methodology outperformed state-of-the-art approaches in terms of segmentation, quantification, and reducing biasing error.

Keywords: distribution patterns; fibroblast cells; HCT-8 colon-cancer cells; nature-inspired techniques; quantification; segmentation

\section{Introduction}

In mimicking the structural and natural complexity of living tissue, current technology such as in vitro 3D spheroid cell culture models is evolving compared to the $2 \mathrm{D}$ cell culture model. In vivo cell growth and cell signaling are highly dependent on the extracellular matrix (ECM) and the interaction produced by different kinds of cells. In vitro 3D cell culture models aid in the study of molecular level tissue function by employing co-culture models and developing drugs for the cancer model in mitigating animal usage for drug testing. In oxygen and nutrients, the gradient can be closely mimicked by 3D cell culture techniques compared to 2D cell culture techniques [1]. However, 3D in vitro spheroid models seriously suffer from image acquisition and standalone image processing algorithms. As a result, user intervention during analysis might lead to heavier biases, thereby leading to erroneous results.

Such issues can be overcome by different automated computer-aided design (CAD) tasks such as segmentation, i.e., the extraction of the region of interest from the images. In the literature, several studies showed multiple usages of automatic segmentation approaches for variable cellular types [2,3]. Al-Kofahi et al. [4] stated that the segmentation 
of cells through automation is an essential step in image cytometry and histometry. M. Sharma et al. [5] proposed a novel non-linear segmentation model to discriminate and quantify living or dead cells. Xing and Yang [6] focused on digital pathology and microscopy image analysis, and extensively reviewed the techniques. Considerable progress was achieved in the past, but algorithms still suffer from biasing and need to boost their accuracy and robustness, consume less time, and self act against the upcoming applications. Color-based segmentation using traditional clustering algorithms, on the other hand, is relatively easy, and complexity is lesser when compared to that of segmentation techniques [7]. It is likewise more relevant for biomedical image segmentation, as the count of clusters is known beforehand. However, traditional clustering techniques suffer from various issues such as being trapped in local optima, and having sensitivity to initial cluster centers and boundary-level constraints [8]. In recent advances, many nature-inspired algorithms arose to solve these clustering problems. The hybridization of nature-inspired algorithms with each other and with traditional clustering techniques was described by Krisna et al. [9], Rana et al. [10], and Chowdhury et al. [11] to resolve clustering issues. However, all of these clustering algorithms need much parameter initialization (Table S1), increasing their complexity and manual intervention. Moreover, incorrect parameter initialization affects the end outcome. To skip the overhead of parameter settings, a nature-inspired algorithm called the teacher learning-based optimization (TLBO) algorithm [12] was proposed.

\section{Material Preparations}

In this study, a poly-di-methyl-siloxane (PDMS) based microwell array chip was utilized to co-culture NIH3T3 and HCT-8 cells in in vitro. A PDMS based microwell array chip was used to construct the tumor spheroids. The well known soft lithography process was used to fabricate the microwells, and fabrication steps were followed as given by Patra et al. [13]. Images were obtained by scanning a horizontal cross-sectional view using fluorescence based confocal microscopy. To make the cells visible under confocal microscopy, NIH3T3 cells were labeled with CellTracker ${ }^{\mathrm{TM}}$ Green CMFDA (5chloromethylfluorescein diacetate) dye (Thermo Fisher Scientific, China). HCT-8 cells were labeled with CellTracker ${ }^{\mathrm{TM}}$ Blue CMHC (4-chloromethyl-7-hydroxycoumarin) dye (Thermo Fisher Scientific, China). Dyes were functionalized as per the manufacturer's instructions. Briefly, both cell types were incubated with the respective cell tracker dye $(50 \mu \mathrm{L})$ for $30 \mathrm{~min}$ at $37^{\circ} \mathrm{C}$. Cells were further washed 3 times with PBS, mixed, and seeded on the microwell array chip for spheroid formation.

HCT-8 and NIH3T3 cells (blue and green stained cells) were co-cultured in different ratios of 2:0.5; 2:1; 2:2; and 2:4, as shown in Figure 1 ; While preparing the in vitro 3D cell spheroid, the spheroid shaping capabilities expanded and became quicker by including more NIH3T3 cells with the HCT-8 cells. To analyze the cellular distribution in the spheroids, 3D images were captured by using confocal microscopy. Z-direction images were captured (using 10x objective) from the bottom of each spheroid with $6 \mu \mathrm{m}$ step sizes of 50 slices, i.e., a total of $300 \mu \mathrm{m}$, which was equal to the spheroid size, as shown in Figure 2.

This study compares the different intra and inter-domain clustering techniques in the clustering of colored cells. The outcome of the best performing algorithms was used to quantify the cells and estimate the densest region of the colon-cancer cells over an in vitro 3D cell spheroid using a novel region estimation algorithm based on a distance transform (DT) technique. 

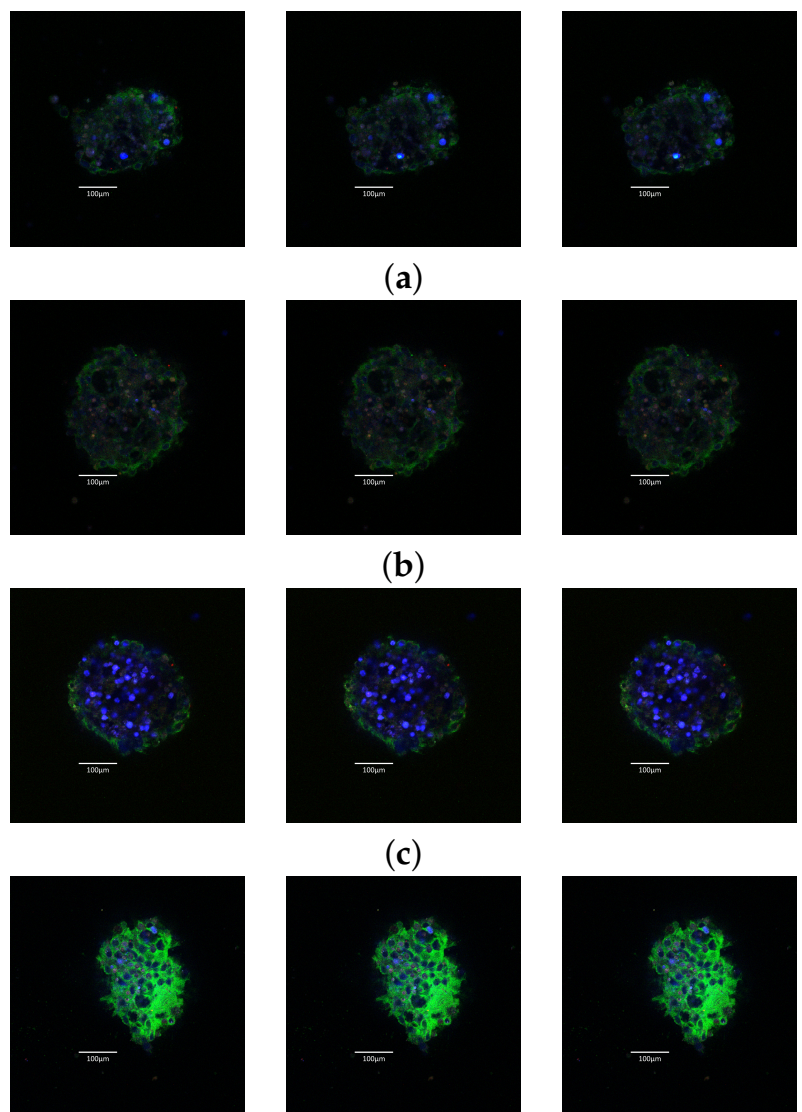

(a)

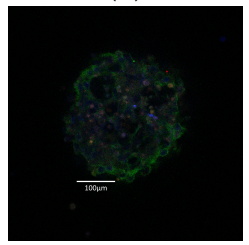

(b)

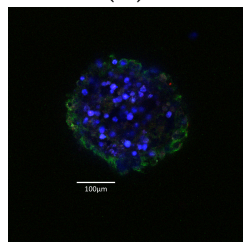

(c)
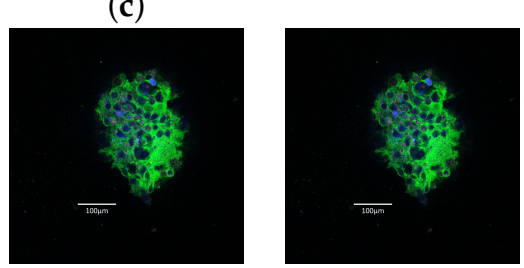

(d)
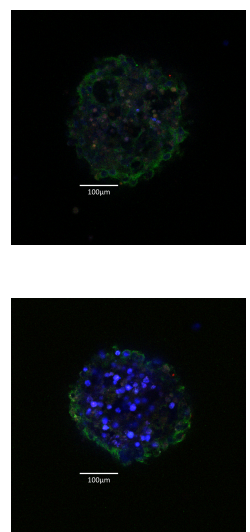

Figure 1. Sample images of each ratio for horizontal cross-section of 3D cell spheroid: (a) 2:0.5; (b) $2: 1 ;$ (c) $2: 2 ;$ (d) $2: 4$.

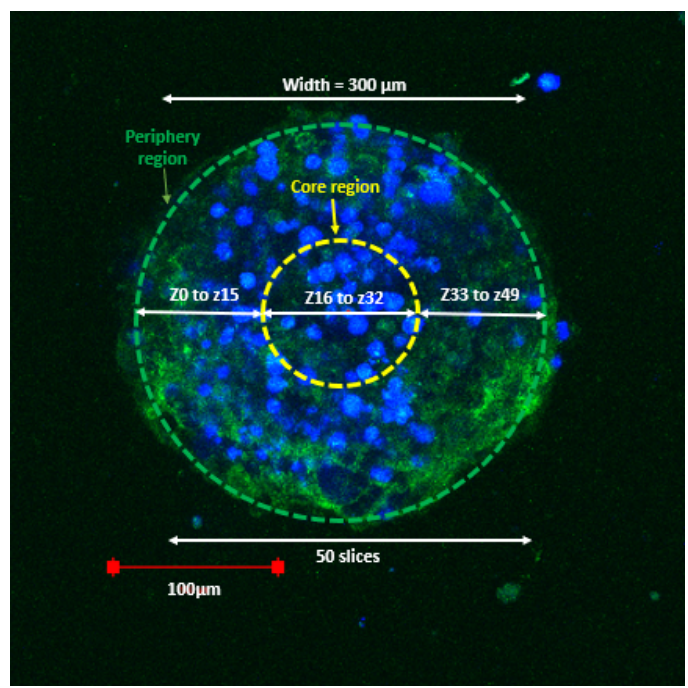

Figure 2. Partitioning of in vitro 3D cell spheroid into regions.

\section{Problem Formulation}

The spheroid was divided into 50 slices $\left(z_{0--49}\right)$ for each ratio (Figure 2). The width of the whole spheroid was $300 \mu \mathrm{m}$, which means that each slice had $6 \mu \mathrm{m}$ thickness. Therefore, the whole in vitro 3D cell spheroid was partitioned into two regions: (1) the core region (from approx. slice nos. $z_{16--32}$ ) and (2) the peripheral region (from approx. slice nos. $\left.z_{0--15} a d_{33--49}\right)$. The densest region was where the concentrations of the HCT-8 cells in the in vitro 3D cell spheroid were the maximum between the two regions. The estimation of the densest region of the colon-cancer cells in the in vitro 3D cell spheroid was 
required for further analysis and treatment planning. With this, the technology of ultralow attachment surfaces of in vitro techniques is of the recent trend $[14,15]$ recently became popular $[16,17]$ in reducing the burden animal model. These microwell technologies have been used for the past few decades [18], and several cancer tumor models were constructed in vitro, which may provide a better environment to build in vitro 3D culturing of patient derived xenografts (PDX). Further improving image processing techniques may advance our understanding of stromal cell distribution in real tumor tissue in vitro, which helps in personalized medicine. Cells have different proliferation rates, but the environment depends on initial cell seeding density in the spheroid. We are currently experimenting on the aspect of the proliferation rate and monitoring them at different time points.

In order to understand the regional distribution of in vitro 3D cell spheroids, the total number $N_{b}$ of HCT- 8 cells and $N_{g}$ NIH3T3 cells present in the in vitro 3D cell spheroid was considered. The cells were considered to be circular. The counts of HCT-8 and NIH3T3 cells were evaluated for horizontal cross sectional images of the in vitro 3D cell spheroid for each slice (slice nos. $\left.z_{0--49}\right)$. The identification of the densest region depends on the maximal concentrations of the HCT- 8 cells in two different regions i.e., core $\left(B_{c}\right)$ and periphery $\left(B_{p}\right)$. Therefore, it was formulated as

$$
R_{\text {dense }}=\left\{\begin{array}{l}
C R, \text { ifCount }\left(B_{c}\right)>\operatorname{Count}\left(G_{p}\right) \\
\| \operatorname{Count}\left(B_{c}\right)>\operatorname{Count}\left(B_{p}\right) \\
P R, \operatorname{ifCount}\left(B_{p}\right)>\operatorname{Count}\left(G_{c}\right) \\
\left.\| \operatorname{Count}\left(B_{p}\right)>\operatorname{Count}\left(B_{c}\right)\right)
\end{array}\right.
$$

where $G_{c}$ and $G_{p}$ are the count of NIH3T3 cells for the core and peripheral regions, respectively; $B_{c}$ and $B_{p}$ are the count of HCT- 8 cells for the core and peripheral regions, respectively. The proposed region estimation algorithm finds the densest region $R_{\text {dense }}$ of the HCT- 8 cells and gives the distribution patterns of the HCT- 8 and NIH3T3 cells from the core to the peripheral region over the in vitro 3D cell spheroid.

\section{Proposed Methodology}

The overall proposed methodology comprises two main steps: (1) foreground cell clustering, and (2) region estimation and quantification, as shown in Figure 3. The original images of the in vitro 3D cell spheroid were first converted from the $R G B$ color space to the $L^{*} a^{*} b^{*}$ color space. Unlike the RGB color model, the $L^{*} a^{*} b^{*}$ color model is approximately close to human vision. This color model provides uniformity in the range of perception [19]. Extracted $a * b *$ components from the RGB images were given as input to the TLBO clustering algorithm. The approach has two resultant phases, the teaching phase and the learner phase, as described in [12]. Initially, a K number of clusters were taken, each cluster datum or pixel datum was defined as a learner, and centroids that were selected randomly for each cluster are called teachers. After that, each learner's Euclidean distance with the centroid for all $\mathrm{k}$ clusters and fitness value was evaluated. Using the learner-phase steps given in [12], each learner was modified. Likewise, the centroids or the best learners and the existing solution are modified. After reaching maximal iterations $I_{\max }$, the foreground cells were separately clustered. The HCT- 8 and NIH3T3 cluster cell images were then converted into a binary image using Ostu's global thresholding technique [20]. 


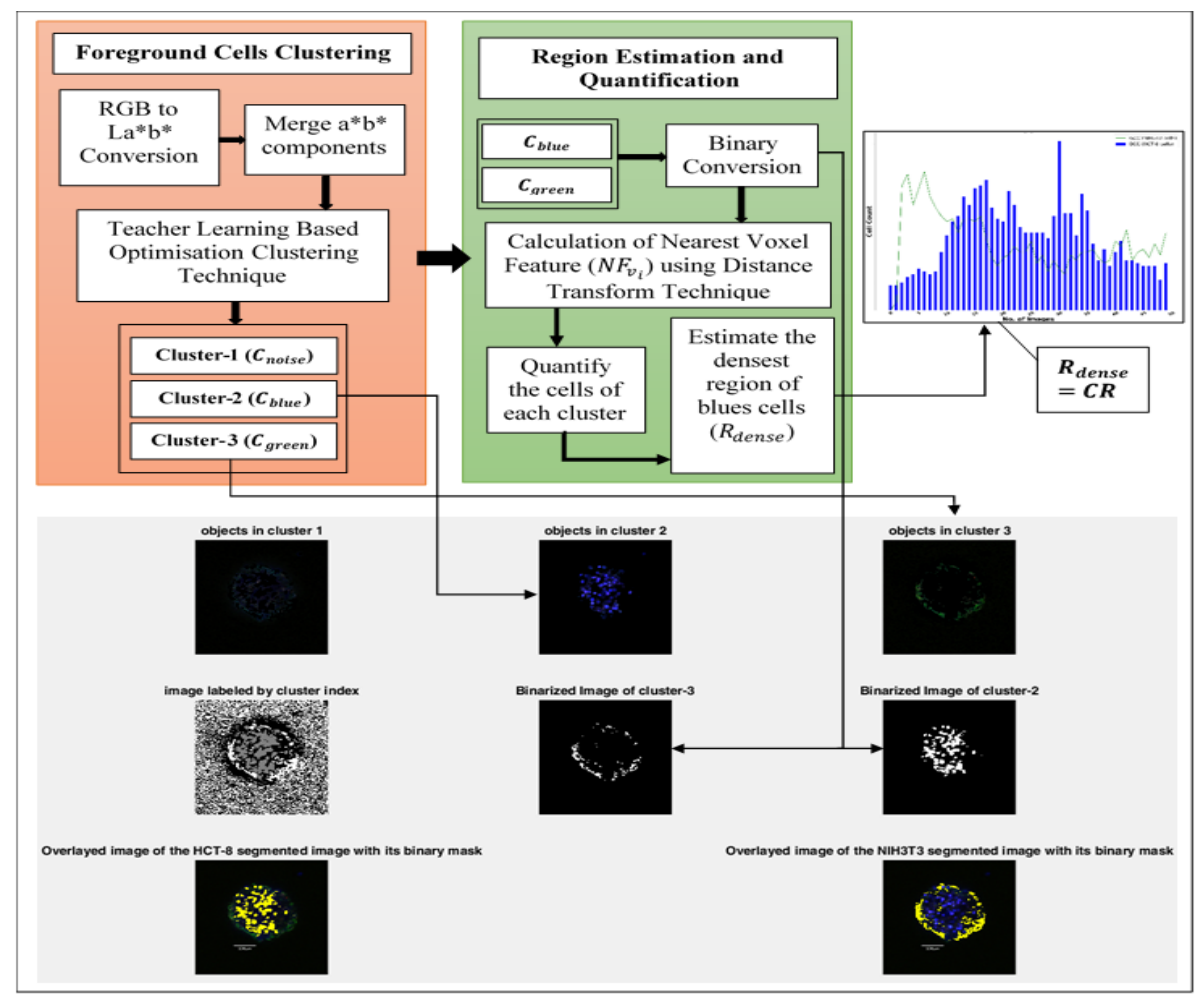

Figure 3. Resultant view of proposed methodology.

Binary images $B_{b}$ and $B_{r}$ were then given as input in Algorithm 1 to estimate the densest HCT- 8 cell region by finding the cell count. The nearest feature voxel $N F_{v_{i}}$ of the $B_{b}$ and $B_{r}$ were calculated as follows:

$$
N F_{v_{i}}= \begin{cases}0 & v_{i} \in B_{v_{i}} \\ \min \left(\left\|v_{i}, v_{j}\right\|\right) & \left(v_{i}, v_{j}\right) \in F_{v_{i}}\end{cases}
$$

where, $\left\|v_{i}, v_{j}\right\|=\left\|v_{i}-v_{i_{0}}, v_{j}-v_{j_{0}}\right\|, \forall\left(v_{i_{0}}, v_{i_{0}}\right) \in B_{v_{i}}$ is any distance metric.

The outcome of DT depends on the selection of distance metrics, which varies from application to application. However, among all other metrics, the Euclidean distance metric was the most generalized because its measurement corresponds to the way in which objects are measured in the real world, and is rotation-invariant. The metric uses the $L_{2}$ norm and is defined as $\left\|v_{i}, v_{j}\right\|_{2}=\sqrt{v_{i}^{2}+v_{j}^{2}}$. After obtaining the nearest feature voxel matrix, maxima propagation is applied using the inverted mask matrix of $N F_{v_{i}}$ and by limiting the propagation using some fraction $\left(c_{1}=0.5\right)$ of the maximal distance of the nearest feature voxel matrix $N F_{v_{i}}$. To control the extent of propagation of $M P_{i}, M P_{i}$ is dilated with the $3 \times 3$ matrix of all ones. This operation transforms the finer distance image into coarser segments. After that, the different numbers of segments or levels as $L v l_{i}$ are found using the "unique" function of MATLAB. Then, background voxels were removed from the $L v l_{i}$ matrix. The area threshold value was calculated to impose the minimal area constraints for segments. After that, the 8-connected component (or blobs or cells) area and pixel indices were found using the "region props()" inbuilt function of MATLAB, which simply sums the pixels of a particular region (area) by using their assigned labels. Then, the area is thresholded by using the extracted areas and finding the count of blobs or cells encountered within that area region. This gave the count of HCT- 8 and NIH3T3 cells in different regions of the in vitro 3D cell spheroid. On the basis of these counts, i.e., $B_{c}, B_{p}, G_{c} a n d G_{p}$ the densest HCT-8 cell region $\left(R_{\text {dense }}\right)$ using Equation (1) was estimated. 


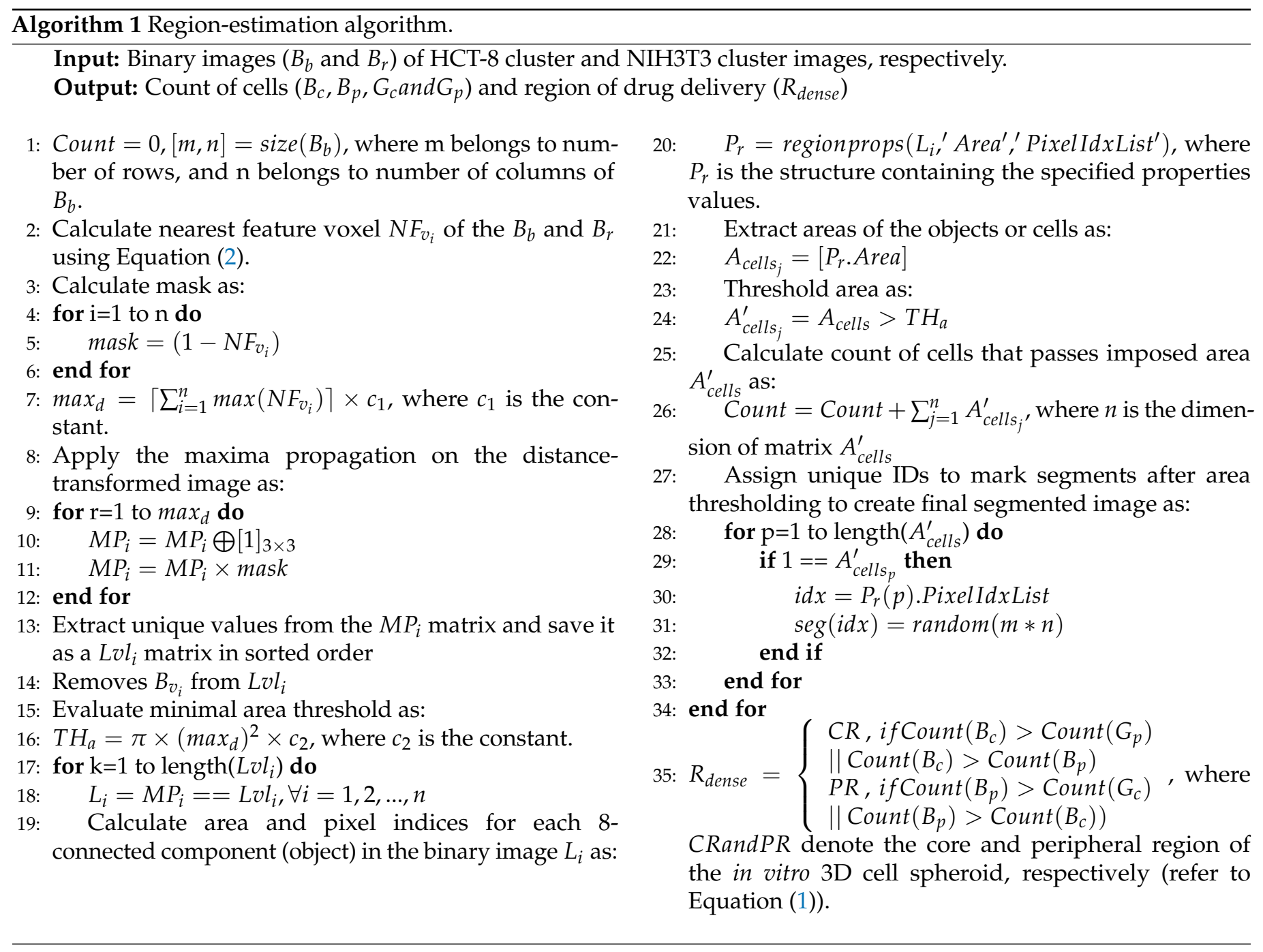

\section{Experiment Analysis}

The proposed methodology was simulated using MATLAB R2017a on a system with Intel 7th generation $4770 @ 3.40 \mathrm{GHz}$, and validated using the dataset as mentioned in Section 1. The dataset was divided into 4 types of ratio images of NIH3T3 and HCT-8 cells. In the experiment analysis to evaluate the proposed methodology's performance, the capability of the foreground cell clustering algorithm (TLBO) was evaluated on the basis of qualitative and quantitative results as described in Section 5.1. Likewise, the region estimation algorithm's ability for cell quantification and in reducing the biasing error was evaluated by comparing it with ImageJ software as described in Section 5.2. All results are shown for 2:0.5 ratio images for reference, and the rest of the ratio images figures, graphs, and tables are provided as supplementary material.

\subsection{Comparative Analysis: Qualitative and Quantitative}

In this section, the reason is provided for selecting the TLBO nature-inspired clustering algorithm to extract the foreground cells of the in vitro 3D cell spheroid. However, comparing the ground truth images of colon-cancer cells was not possible due to the unavailability of segmentation masks to compare them with the TLBO clustering segmentation results. Therefore, the TLBO clustering algorithm results were compared with other nature-inspired clustering and traditional clustering approaches: Particle Swarm Optimization (PSO), Genetic Algorithm (GA), Invasive Weed Optimization (IWO), k-means (KM), k-medoids (KMed), and Fuzzy C-means (FCM). Results were compared on the basis of qualitative and 
quantitative results. On 200 iterations, the value of the fitness metrics (clustering cost as shown in Equation (3)) converged, as shown in Figure 4. Thus, all clustering experiments were performed by running the algorithms on 200 iterations. The parameter values used for each algorithm are shown in Table S1 (provided in the supplementary material).

$$
\begin{gathered}
\operatorname{Cost}_{j}=\frac{\sum_{k=1}^{n_{\max }}\left(d_{k}\right)}{n_{\max }} \\
d_{k}=\sqrt{\left(x_{s}-y_{t}\right)^{2}+\left(x_{s}-y_{t}\right)^{2}}
\end{gathered}
$$

$\forall s, t \in 1,2, \ldots, C_{n}$, where $n_{\max }$ is the number of distances $d_{k}$ calculated within the clusters.

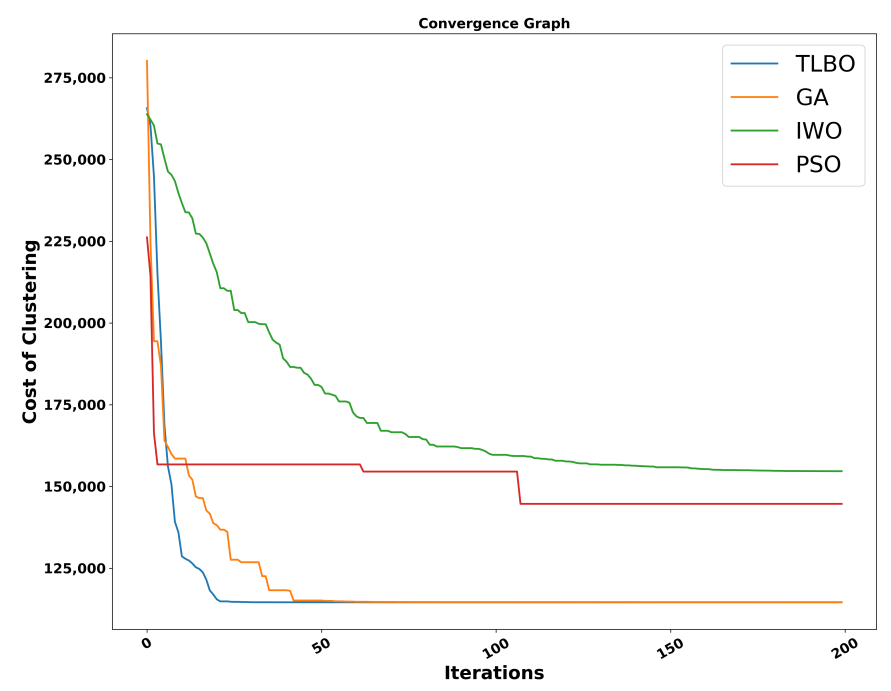

Figure 4. Cost of clustering graph of each algorithm to show convergence up to 200 iterations.

A qualitative comparison among clustering algorithms is shown in Tables 1-3 for the cellular ratio of 2:0.5 using three different images. Different cellular ratios of 2:1, 2:2, and 2:4 are shown in Tables S2-S4, respectively. The tables' information contains labeled images, the three clusters, and the graphical representation of clusters and their centroids. In the clustering of HCT-8 cells, the TLBO algorithm had greater potential than that of other algorithms. A similar phenomenon was observed for other cellular ratios, as shown in Tables S2-S4. In the quantitative comparison, all clustering algorithms were compared on the basis of quantization error (QE) [21] and best cost (BC) (Equation (3)). The quantization error of 2:0.5 ratio images is shown in Figure $5 \mathrm{a}, \mathrm{b}$, and the best cost is shown in Figure $5 \mathrm{c}, \mathrm{d}$. The different cellular ratios of quantization error and best cost are shown in Figures S5-S10.

Qualitative and quantitative analysis suggested that TLBO clustering performance was better than that of other nature-inspired clustering approaches (PSO, GA, IWO) and traditional clustering approaches (k-means, k-medoids, FCM). Therefore, the TLBO clustering algorithm was selected for foreground cell clustering in the proposed methodology. 


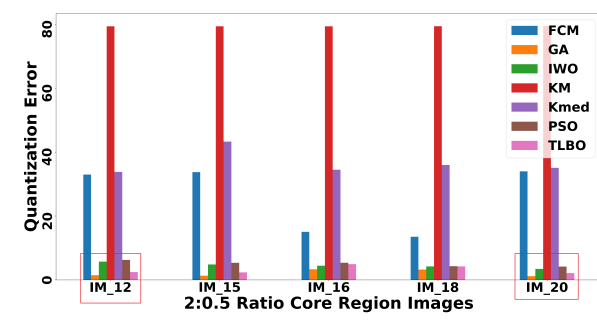

(a)

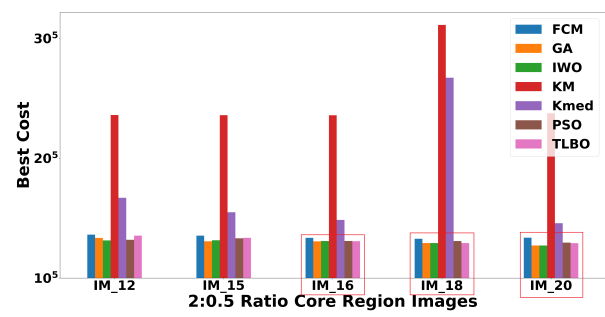

(c)

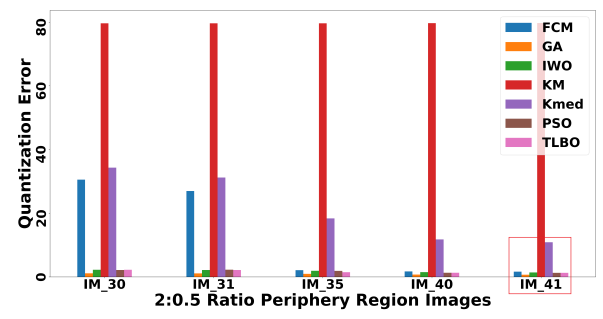

(b)

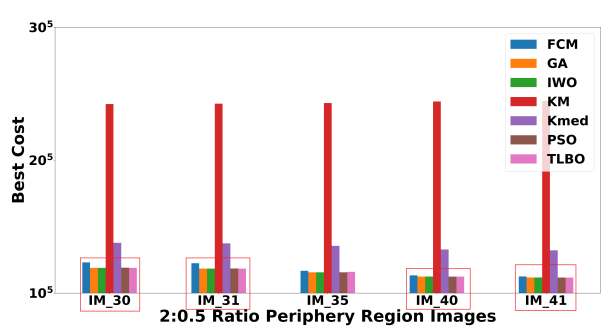

(d)

Figure 5. Comparative analysis between teacher learning based optimization (TLBO) and other existing nature-inspired clustering techniques using 2:0.5 ratio slices based on (a,b) quantization error (QE) and (c,d) best cost (BC).

Table 1. Comparative Analysis of visual results of TLBO with other clustering algorithms for 2:0.5 ratio image of Figure 1a.

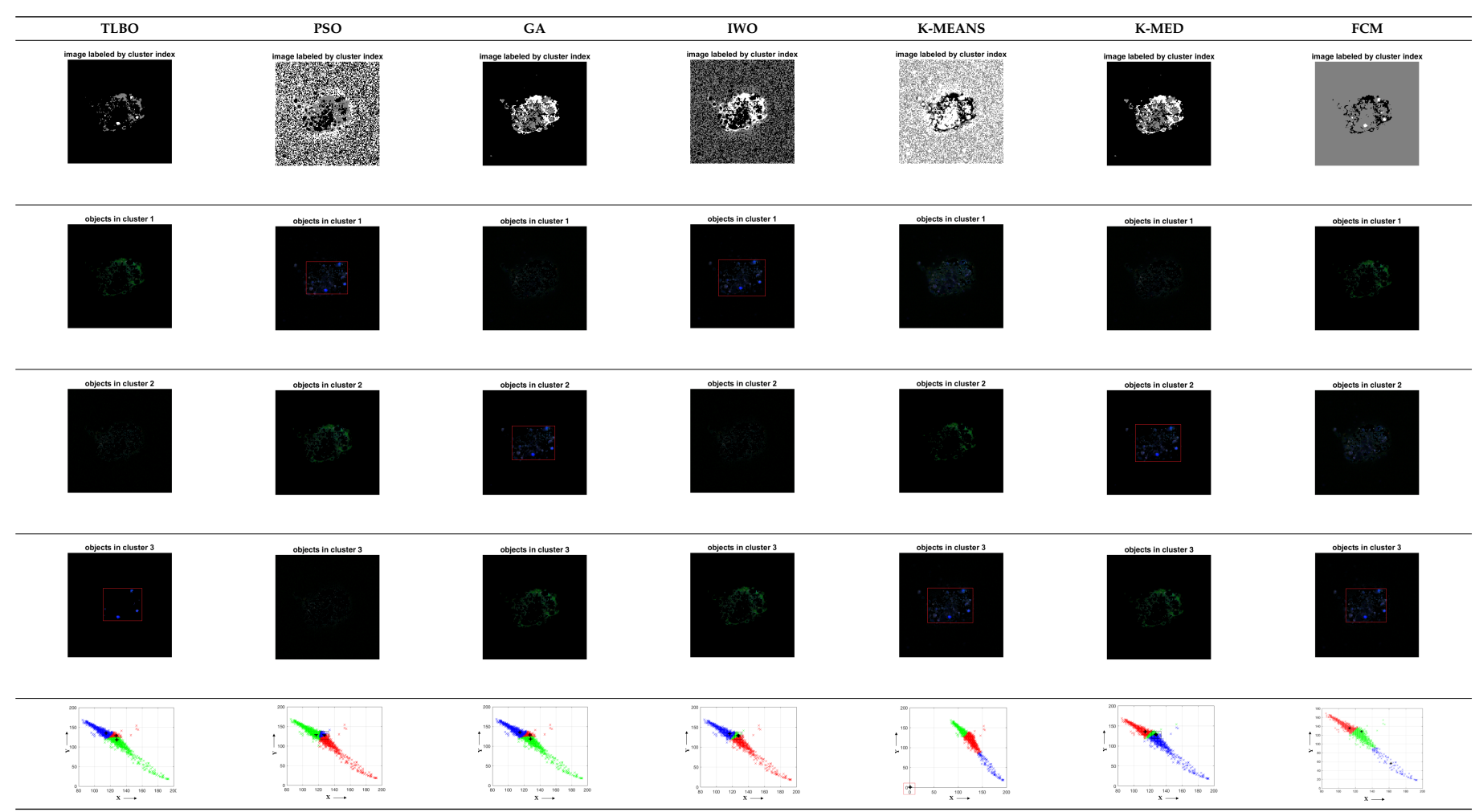


Table 2. Comparative analysis of visual TLBO results with other clustering algorithms for 2:0.5 ratio image of Figure 1a.

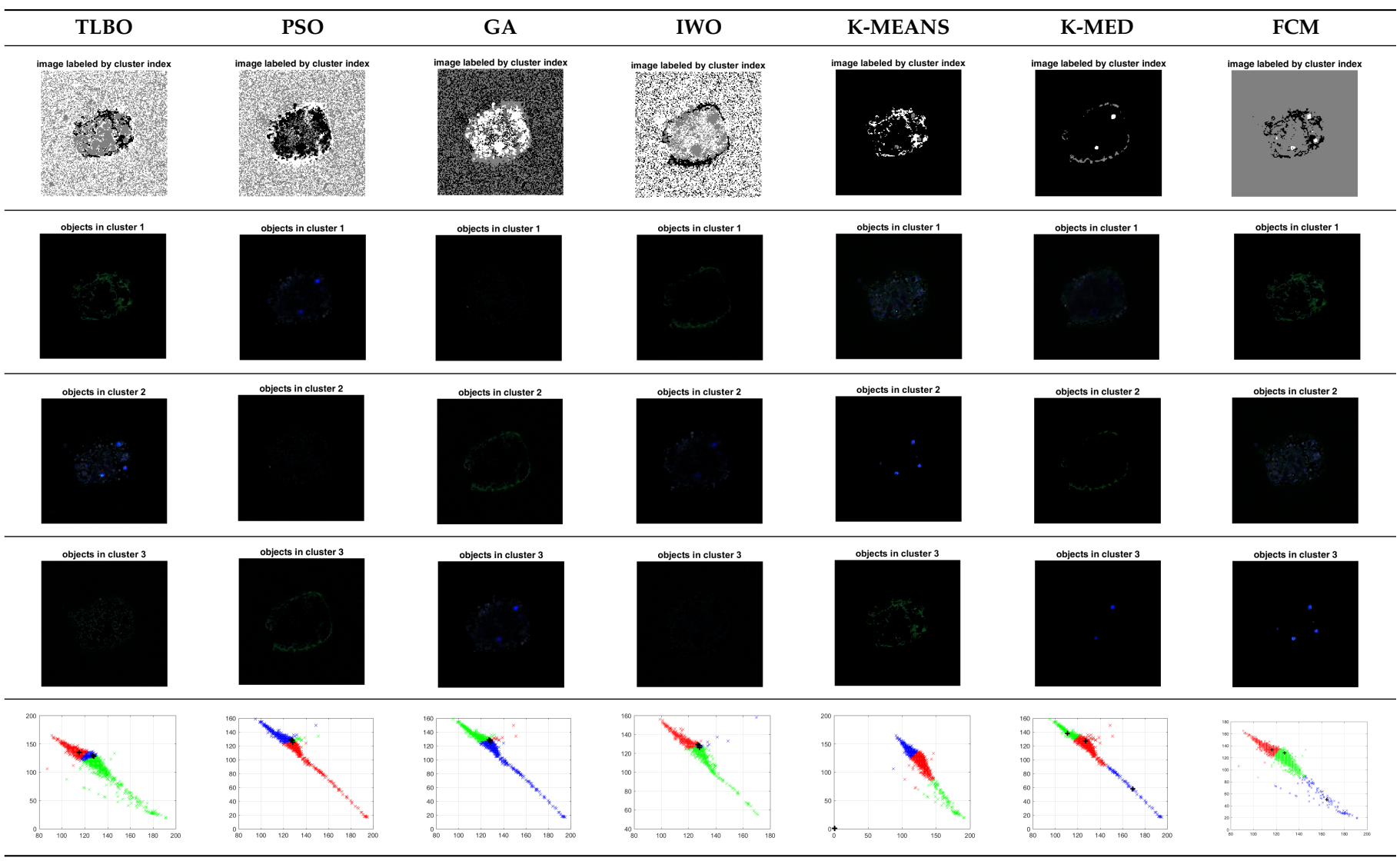

Table 3. Comparative analysis of visual TLBO results with other clustering algorithms for 2:0.5 ratio image of Figure 1a.

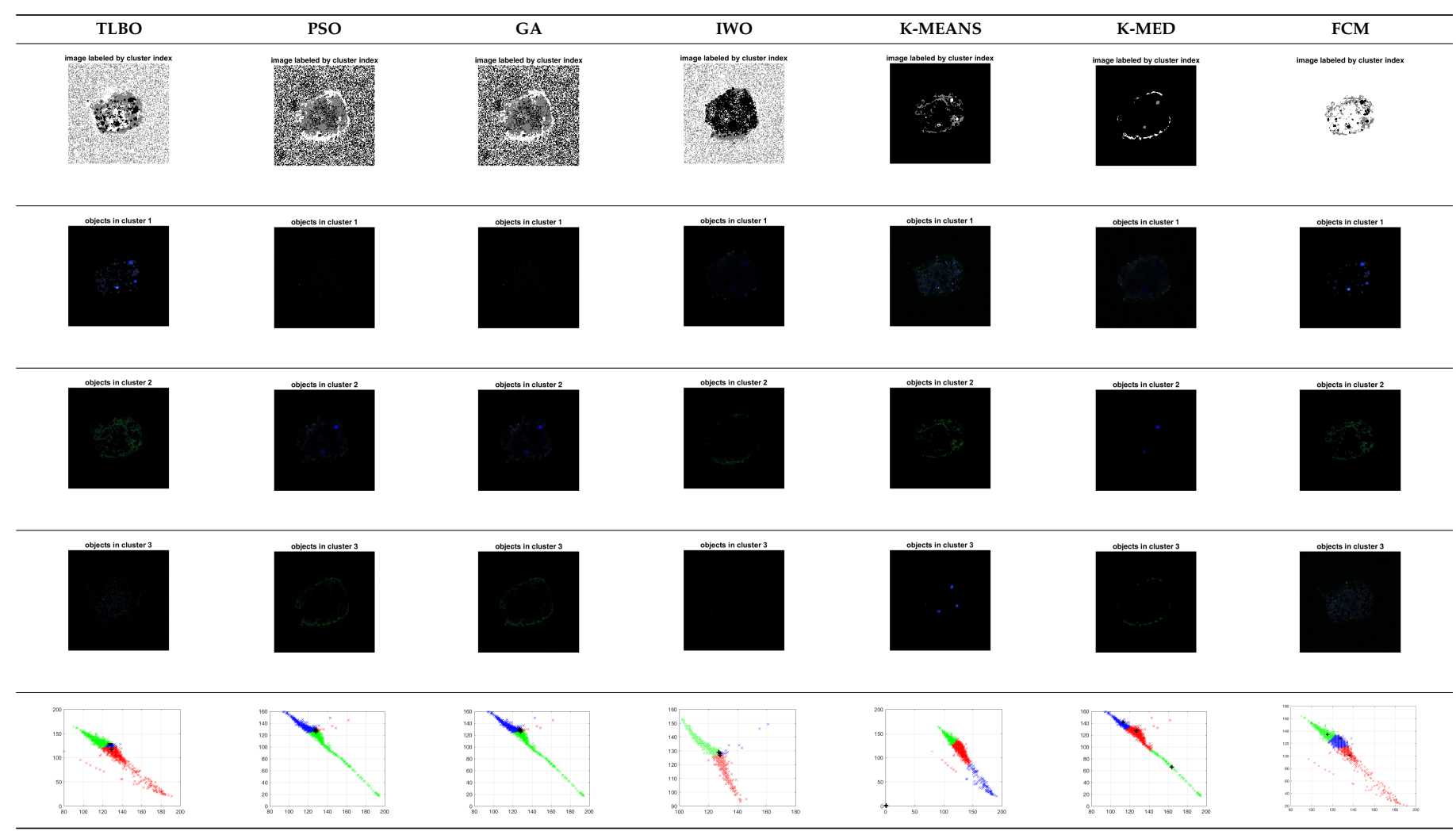




\subsection{Quantitative Results of Region-Estimation Algorithm}

In cell quantification, a thresholding process was employed. During the start of processing or in the optimization threshold, images were inspected or visualized manually, and the best set of images (in this case, 2:1) that were visualized were taken in optimizing the threshold value. This might be because of variability in laser power excitation during the acquisition of images, and intensity based image variations had minimal or no effect on the quantification of cellular analysis. Employing one ratio threshold to other cellular ratios did not affect the results, as cellular sizes were uniform in all cellular ratios. Fibroblast (NIH3T3) cell-to-cell interactions are tight and they form tight junctions; therefore, cell boundaries may not be distinguishable. NIH3T3 cells were clustered, and various thresholding values were applied to find the optimal threshold values, as shown in Figure 6. Figure 6 indicates the segmented NIH3T3 cells overlain with binary masks to evaluate the most relevant threshold value. Values were obtained by multiplying a scalar quantity with maximal intensity value in the image, and passing the binary thresholding data. It was important to choose an optimal threshold value because, by choosing a higher threshold value, intensities start merging into another, and for small threshold values, tiny cells seem to appear that are oversegmented. By visual inspection, and from Figure 6 and 7, the value of $\mathrm{T}=0.125$ corresponded to the best possible results for all image slices.

In this way, clustered NIH3T3 cells were converted into their binary masks using the optimal threshold. The binarized mask was then fed into the RE algorithm 1 to count the NIH3T3 cells. The results of the overlain images of HCT- 8 and NIH3T3 binary masks on its original images are shown in Figure 8.

The distribution of NIH3T3 and HCT-8 cells was as shown in Figure 9a,b for horizontal cross-sectional views. Figure 9a shows the blue (HCT-8) cell count (BCC), and green (NIH3T3) cell count (GCC); and Figure 9b shows the blue (HCT-8) cell area (BCA) and green (NIH3T3) cell area (GCA) of the in vitro 3D cell spheroid for 2:0.5 ratio images. For other cellular ratios, the distribution pattern is shown in Figures S11-S13. Cell-count distribution gave better observation for HCT- 8 cells, whereas the area plot gave better distribution pattern analysis for NIH3T3 cells. The limitation in NIH3T3 cell count can be attributed to the high overlap and the staining procedure of the green cell tracker. After evaluating the count for HCT-8 and NIH3T3 cells (as shown in Figure 9a,b), further evaluation for the region-estimation algorithm (Algorithm 1) was performed for each ratio image. The concentration of HCT-8 cells was the maximum in the core region compared to the whole in vitro 3D cell spheroid. The densest region of HCT-8 cells in the in vitro 3D cell spheroid estimated by the RE algorithm 1 ( $R_{\text {dense }}$ ) was the core region (CR), and the width of the core region was estimated to approximately be $100 \mu \mathrm{m}$. 

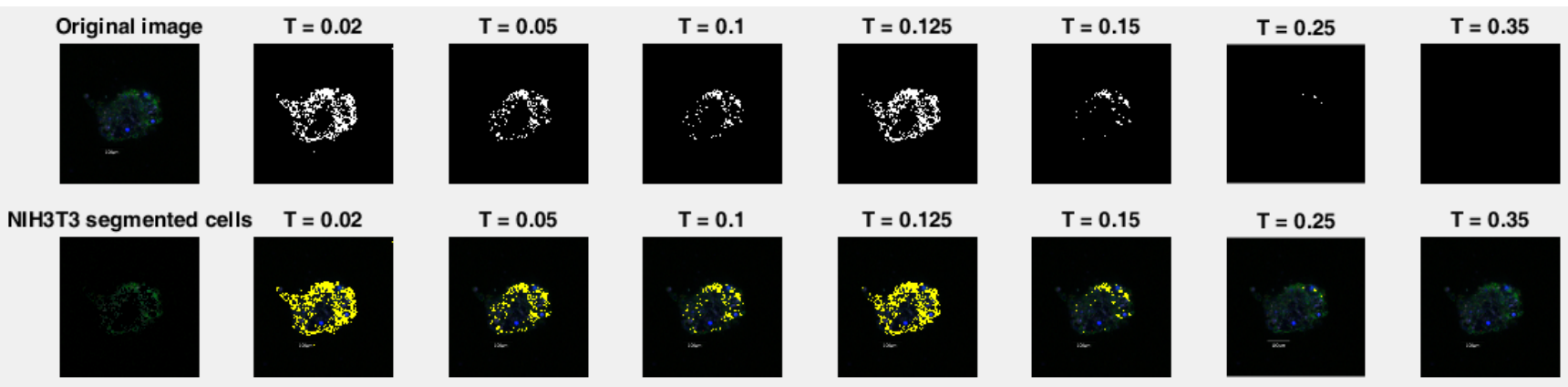

(a)
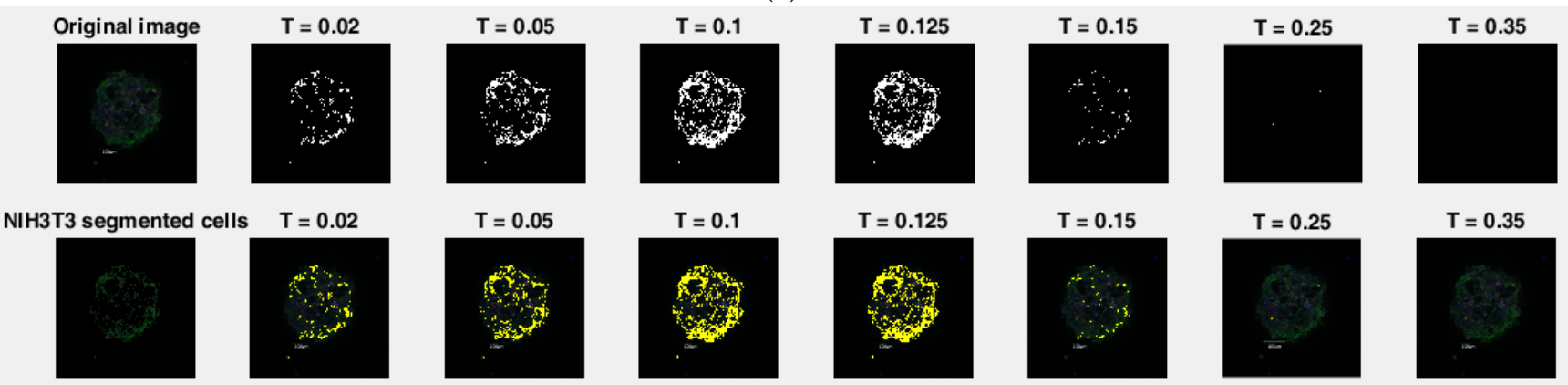

$\mathbf{T}=0.35$

(b)
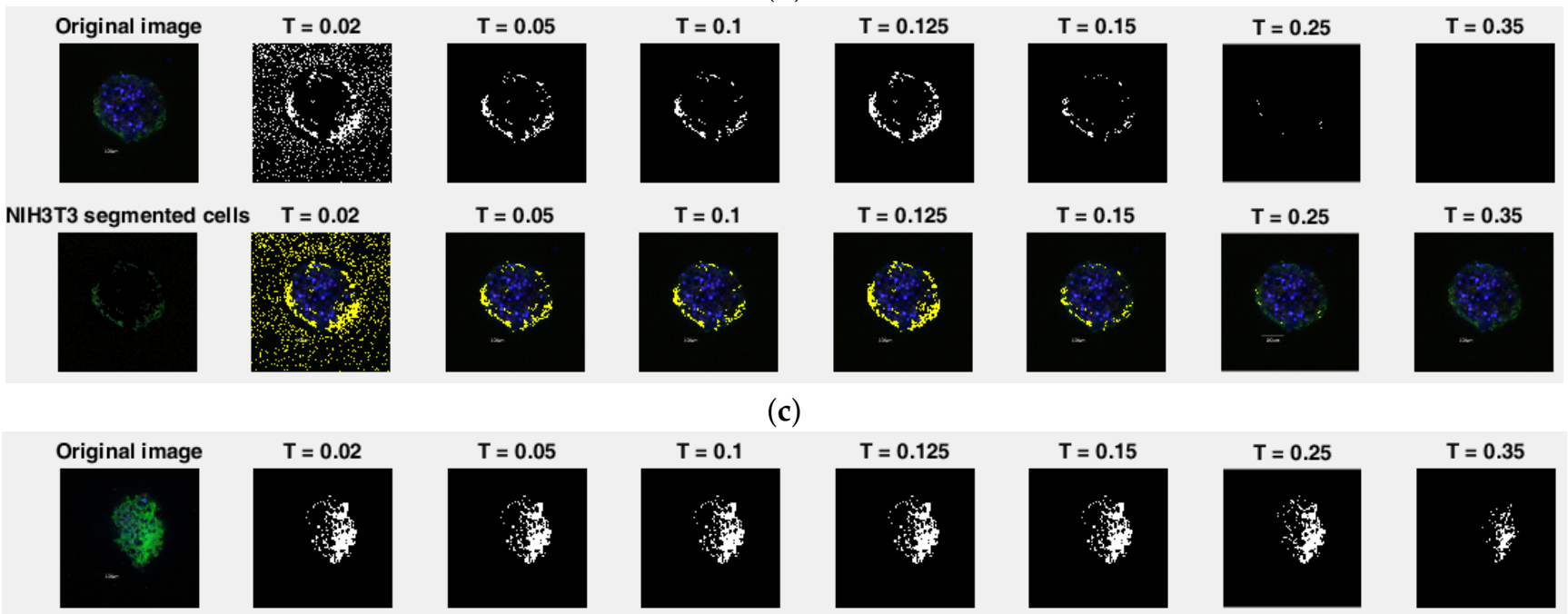

NIH3T3 segmented cells
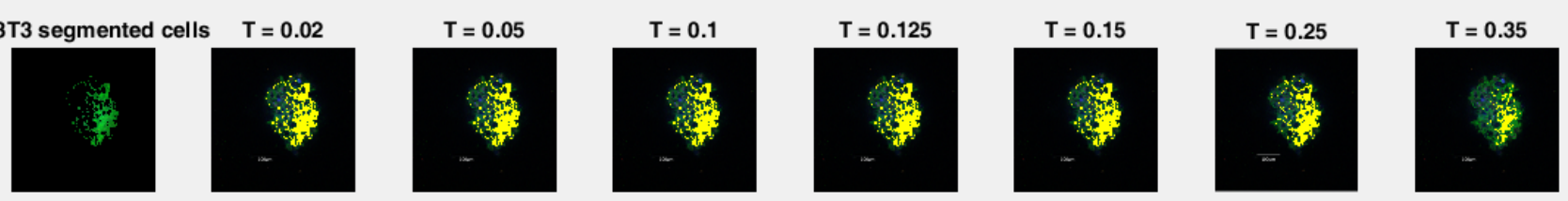

(d)

Figure 6. Optimal threshold evaluation for NIH3T3 cells using various threshold segmentation results. (a) 2:0.5 (2_05_z12), (b) 2:1 (2_1_z14), (c) 2:2 (2_2_z17), and (d) 2:4 (2_4_z10) ratio images. 


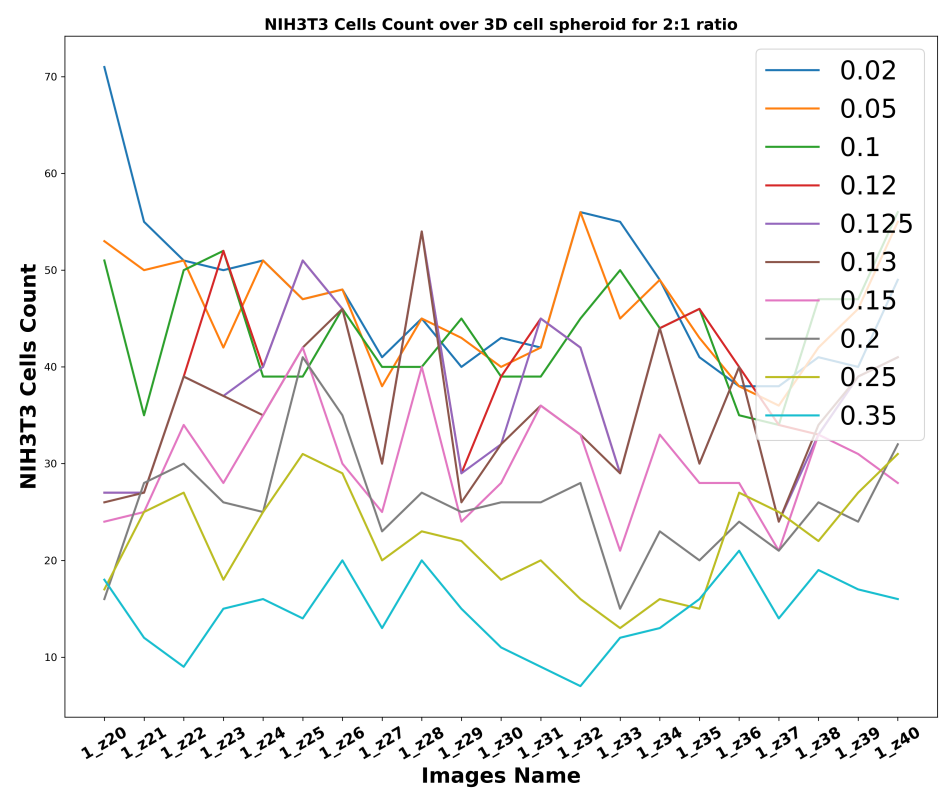

Figure 7. NIH3T3 cells count for 2:1 ratio of horizontal cross-sectional images for various threshold values.

Standalone image analysis software (Image J) was used in comparing the cellular quantification of the results. The procedure to segment and quantify HCT- 8 and NIH3T3 cells for all cellular ratio images by Image software was as shown in Figure 10, and the corresponding quantification data was as shown in Figure 11. The process steps during quantification using ImageJ introduce manual biasing: segmentation, thresholding, pixel size, and circularity bias. Briefly, the quantification process was as follows. Slices were input to the ImageJ software, and images were split into three channels: red, green, and blue. After splitting the channels, thresholding was adjusted. A watershed algorithm was then used for segmentation. After segmentation, images were analyzed by providing the pixel size (30-infinity) and circularity (0.30-1.00). Most biases were eliminated during the TLBO clustering and quantification approach except for thresholding bias. The ImageJ software separately clustered the HCT-8 and NIH3T3 cells along with background noise. Though it was required to perform the segmentation algorithm in both approaches, the watershed algorithm was applied in the ImageJ software, whereas TLBO clustering was applied in the proposed methodology. However, the watershed segmentation technique had the drawback of having excessive oversegmentation [22]. In contrast, TLBO separately clustered the HCT-8 and NIH3T3 cells without any background pixels.

Comparative analysis of GCC and BCC using the proposed methodology with the counts of both types of cells obtained from ImageJ software [14] from the peripheral to the core region (slice no. $z_{0--49}$ ) for 2:0.5 ratio images is shown in Figure 12. Other cellular ratios are presented in Figures S14-S16. Because of circularity and pixel-size bias, there was much observable difference between the BCC and GCC of ImageJ software, and the BCC and GCC obtained after applying the proposed methodology. The counting of cells by the proposed methodology showed that most HCT-8 cells were concentrated towards the core region, whereas NIH3T3 cells were more concentrated towards the peripheral region. The difference between manual cell counting and the proposed methodology was approximately $35 \%, 40 \%, 60 \%$, and $80 \%$ for 2:05, 2:01, 2:2, and 2:4, respectively. Moreover, the proposed methodology accurately measured the physiological approximation of the cell count for all cell-ratio processes [15]. The difference percentage of the counting results of each ratio was variate, from $35 \%$ to $80 \%$. This may be due to two reasons:

1. Figure 11 shows that the segmentation results of the ImageJ software for the HCT-8 and NIH3T3 cell clusters also included background pixels (noise). This happened for 
each ratio image, which were further processed and detected (or counted) as blobs (or cells) by the software.

2. Moreover, the ImageJ software needed some parameter adjustment (threshold value, circularity, and size) for segmenting and counting the cells. Biasing error affected the final results.

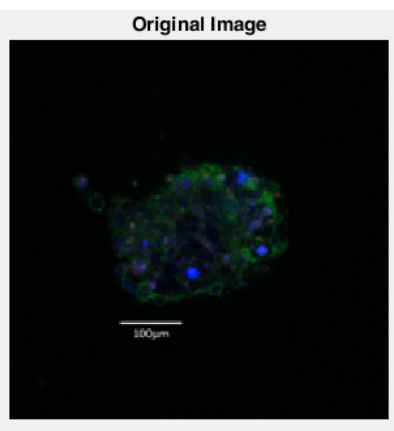

Original Image

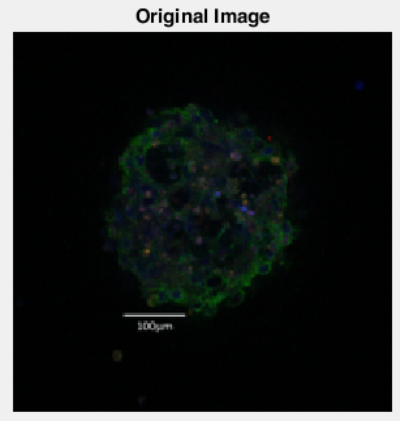

Original Image

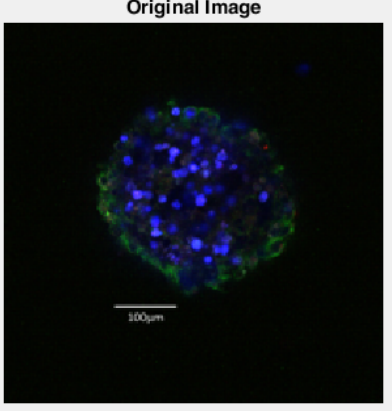

Original Image

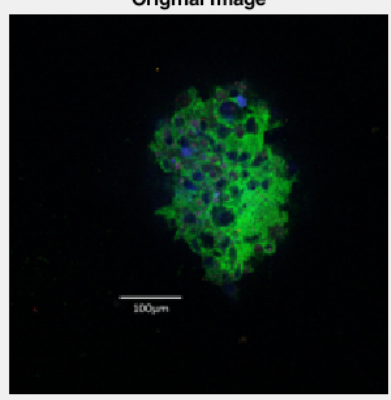

Overlayed image for HCT-8 cells

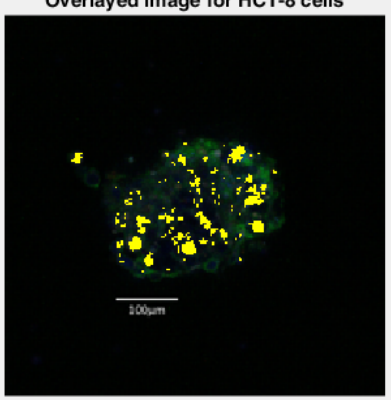

(a)

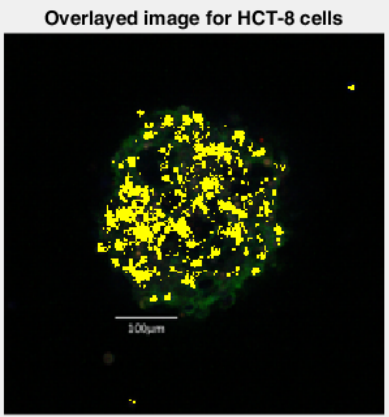

(b)

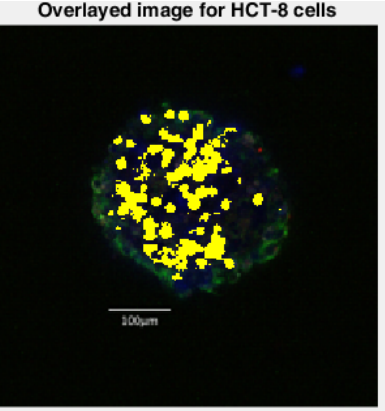

(c)

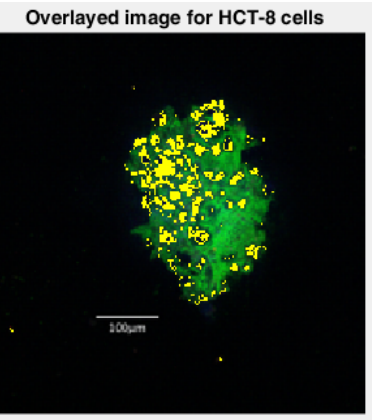

(d)

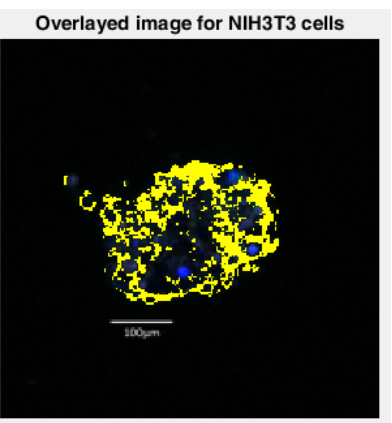

Overlayed image for NIH3T3 cells

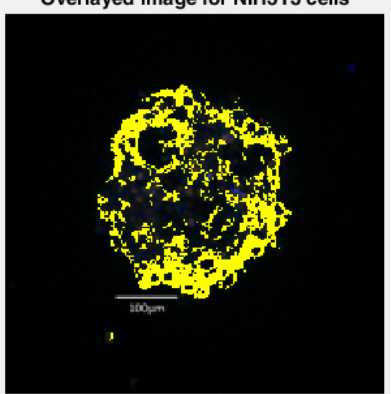

Overlayed image for NIH3T3 cells

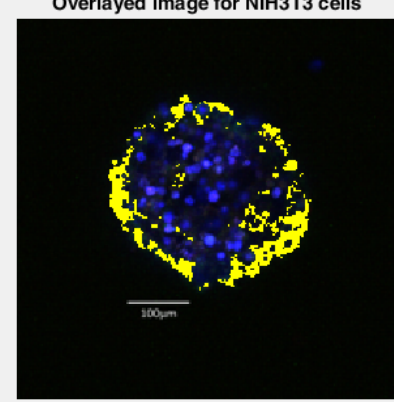

Overlayed image for NIH3T3 cells

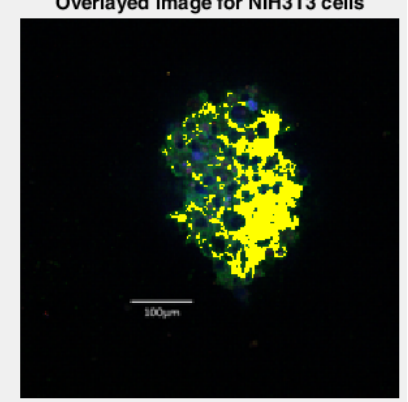

Figure 8. Overlain cluster and original images for HCT-8 and NIH3T3 cells: (a) 2:0.5 (2_05_z12), (b) 2:1 (2_1_z14), (c) 2:2 $\left(2 \_2 \_z 17\right)$, and (d) 2:4 (2_4_z10) ratio images. 


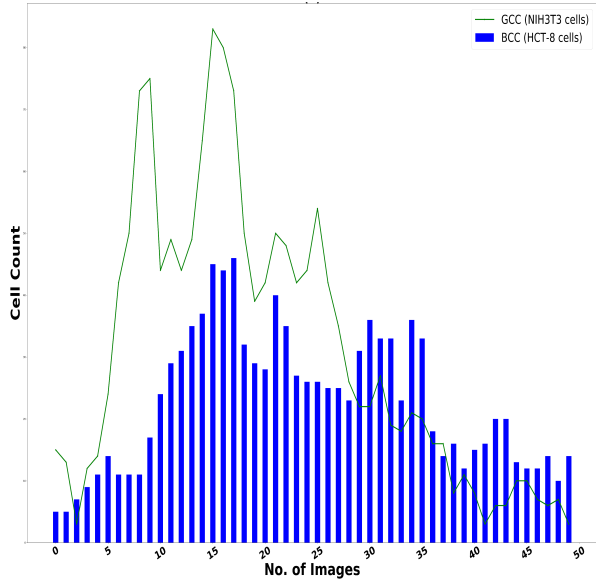

(a)

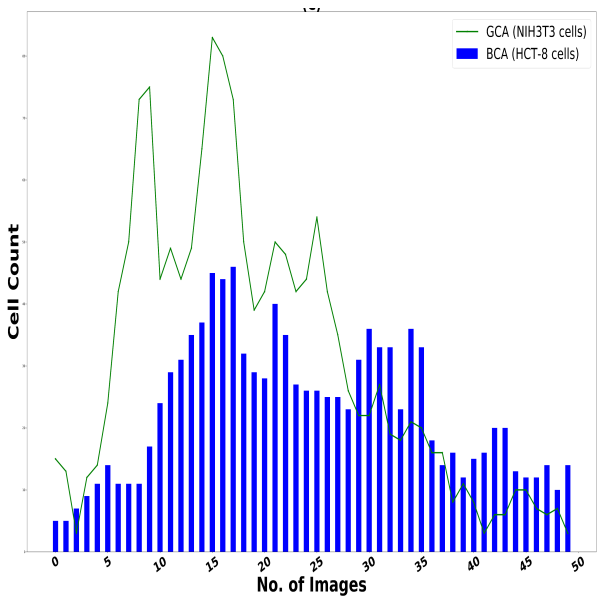

(b)

Figure 9. (a) Blue cell count (BCC) and green cell count (GCC) plots. (b) Blue cell area (BCA) and green cell area (GCA) from periphery to core of in vitro 3D cell spheroid of 2:0.5 ratio slices.

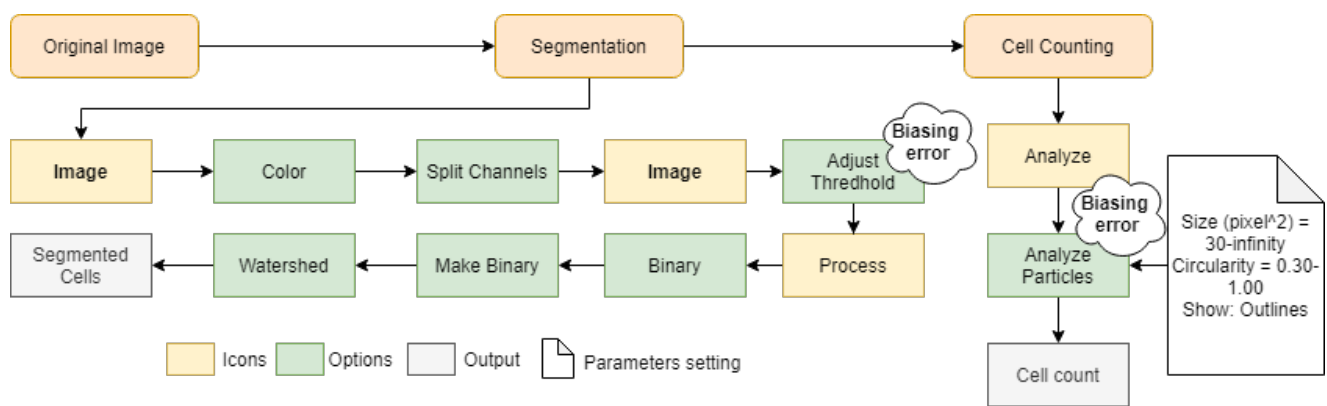

Figure 10. Schematic diagram of procedure followed by ImageJ software for segmentation and quantification of HCT-8 and NIH3T3 cells.

The equation used to evaluate the percentage difference between manual cell counting and proposed-methodology counting is as follows:

$$
\operatorname{Diff}(\%)=\frac{\sum_{i=1}^{N} M_{i}-\sum_{i=1}^{N} P_{i}}{T_{\mathcal{C}}} \times 100
$$

where $\sum_{i=1}^{N} M_{i}$ is the sum of the manual cell count $\forall i=1$ to $N$, and $N$ is the number of slices. Similarly, $\sum_{i=1}^{N} P_{i}$ is the sum of the proposed methodology cell count $\forall i=1$ to N. $T_{\mathcal{C}}$ is the total number of cells present in the in vitro 3D cell spheroid; $N=50$.

Preliminary data derived from the current nature-inspired clustering algorithm (TLBO) help to understand $3 \mathrm{D}$ in vitro systems by the spectrometric location of the extracellular matrix generating protein. Thus, it aids biological scientists in further targeted molecular studies such as polymerase chain reaction (PCR) and Western blot techniques, which are highly selected for treatment planning and diagnostic procedures [23]. Hence, the proposed methodology offers better distribution analysis of HCT-8 and NIH3T3 cells compared to the ImageJ software. 
Original Image

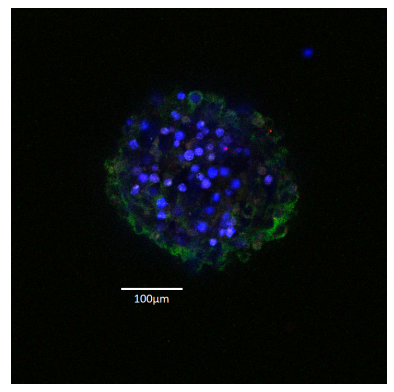

Proposed-Approach Results

ImageJ Results
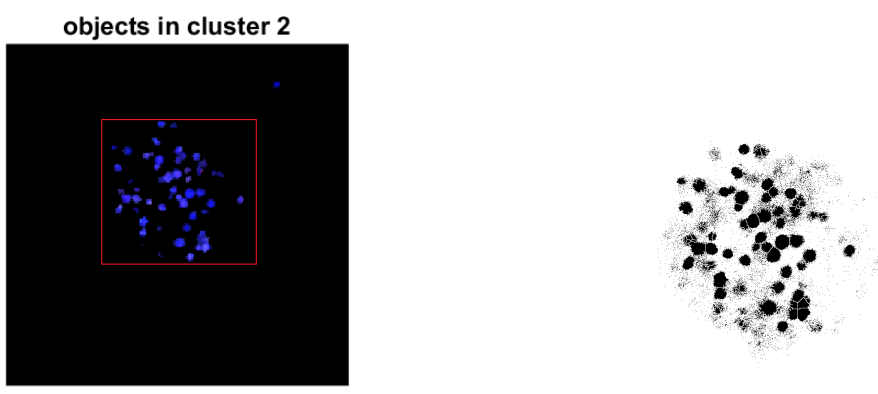

objects in cluster 3
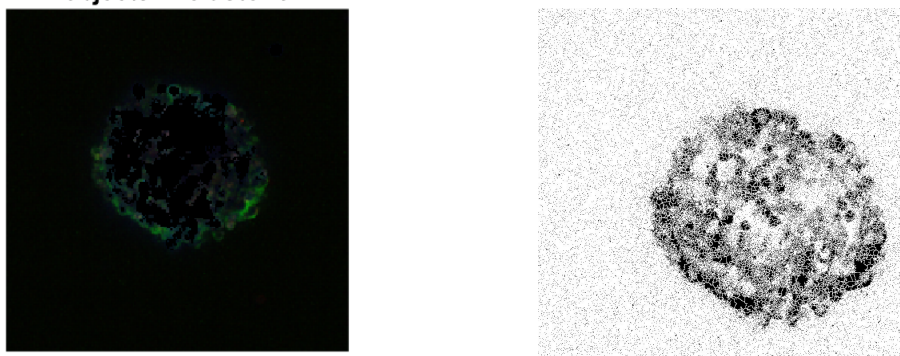

objects in cluster 3
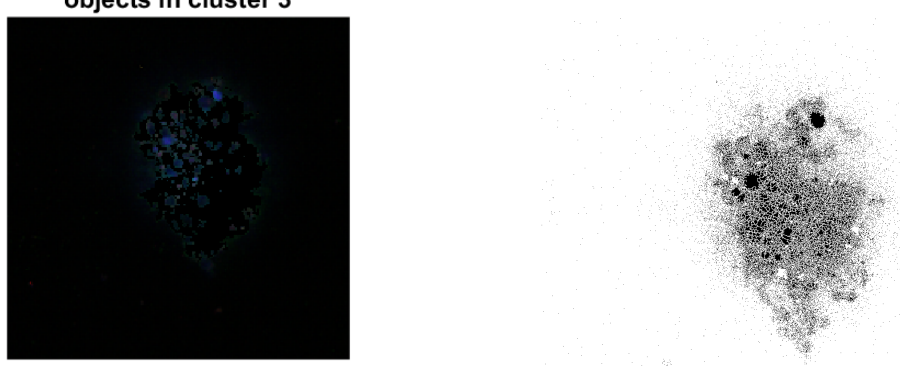

objects in cluster 2
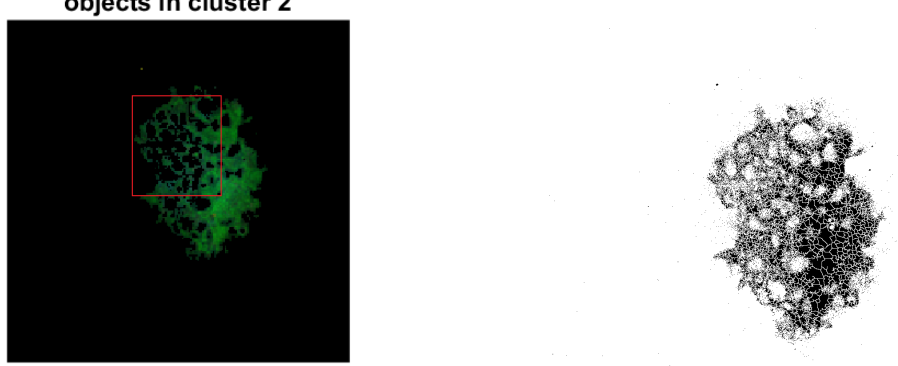

Figure 11. Comparison of TLBO algorithm and ImageJ software based on segmentation of HCT-8 and NIH3T3 cell results. 

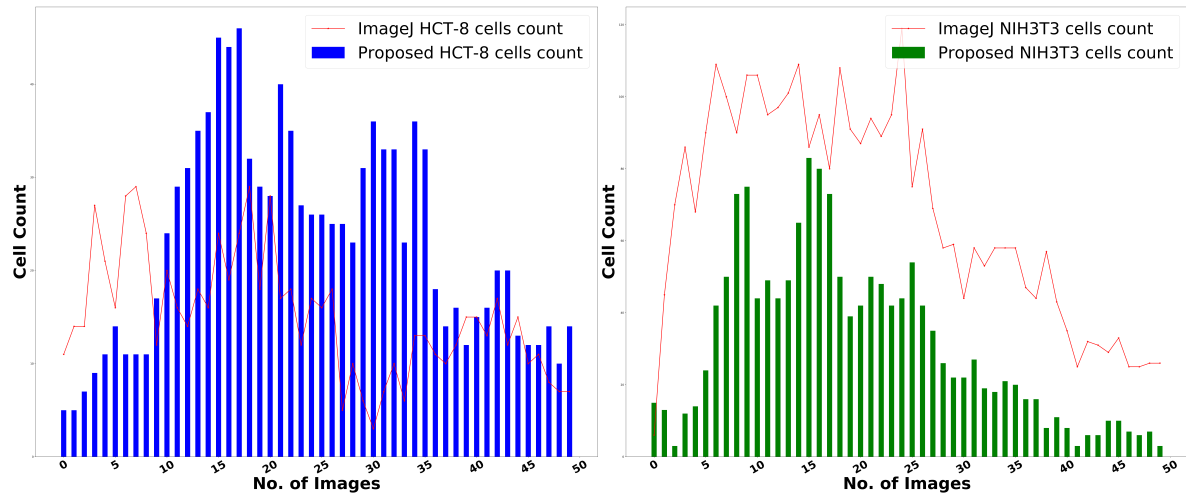

Figure 12. BCC and GCC comparison between ImageJ software and proposed methodology from periphery to core of $3 \mathrm{D}$ cell spheroid for 2:0.5 ratio images.

\section{Conclusions}

The overall proposed methodology analyzes colon-cancer cells' distribution patterns and fibroblast cells in in vitro 3D cell spheroids. On the basis of comparative analysis, the TLBO clustering algorithm was best suited for the cells' color-based segmentation. The region estimation algorithm estimates the densest region of HCT-8 cells in in vitro 3D cell spheroids on the basis of cell quantification. Compared to manual segmentation and quantification by ImageJ software, the proposed methodology reduced the biasing error for cell quantification. The current acquisition methodology was based on and majorly limited by fluorescent trackers and confocal characterization techniques. The possibility of findings can be improved by employing transfecting cells using fluorescent proteins rather than mere cell trackers, and advanced microscopy techniques such as light-sheet microscopy. So, this results in a fast acquisition process and preserves cellular 3D co-cultural spheroids dynamic nature. This enhances the efficient utilization of the distance transform technique and the nature-inspired clustering algorithm. Therefore, one can extend the work by removing the biasing problem of the proposed methodology in quantifying NIH3T3 cells and thresholding the biasing effect.

Supplementary Materials: The following are available online at https:/ / www.mdpi.com/2076-341 $7 / 11 / 10 / 4636 / s 1$.

Author Contributions: Conceptualization, methodology, validation, writing-original-draft preparation: M.S.; data curation, conceptualization, formal analysis, visualization, writing-review and editing: V.S.G. and M.P.K.; funding acquisition, supervision: F.G.T.; and supervision and resources: M.B. All authors have read and agreed to the published version of the manuscript.

Funding: This research was funded by the Ministry of Science and Technology (MOST) 108-2638-E007-001-MY2.

Institutional Review Board Statement: Not applicable.

Informed Consent Statement: Not applicable.

Data Availability Statement: Not applicable.

Acknowledgments: The authors would like to thank the ABV-Indian Institute of Information Technology and Management, India for providing the opportunity for the coordination of research work with researchers of National Tsing Hua University, Taiwan, ROC. The authors also acknowledge with thanks ABV-IIITM and NTHU for providing all facilities and expenditures related to the experiment setup, laboratory equipment, computational platform, and other requirements for the entire study and documentation of the work.

Conflicts of Interest: The authors declare no conflict of interest. 


\section{References}

1. Koduri, M.P.S.; Goudar, V.; Shao, Y.W.; Hunt, J.A.; Henstock, J.R.; Curran, J.; Tseng, F.G. Fluorescence-based nano-oxygen particles for spatiometric monitoring of cell physiological conditions. ACS Appl. Mater. Interfaces 2018, 10, 30163-30171. [CrossRef]

2. Yang, H.; Ahuja, N. Automatic segmentation of granular objects in images: Combining local density clustering and gradientbarrier watershed. Pattern Recognit. 2014, 47, 2266-2279. [CrossRef]

3. Sharma, M.; Bhattacharya, M. Segmentation of CA3 Hippocampal Region of Rat Brain Cells Images Based on Bio-inspired Clustering Technique. In Proceedings of the 2019 IEEE International Conference on Bioinformatics and Biomedicine (BIBM), San Diego, CA, USA, 18-21 November 2019; pp. 2438-2445.

4. Al-Kofahi, Y.; Lassoued, W.; Lee, W.; Roysam, B. Improved automatic detection and segmentation of cell nuclei in histopathology images. IEEE Trans. Biomed. Eng. 2009, 57, 841-852. [CrossRef]

5. Sharma, M.; Bhattacharya, M. Discrimination and quantification of live/dead rat brain cells using a non-linear segmentation model. Med. Biol. Eng. Comput. 2020, 1-20. [CrossRef] [PubMed]

6. Xing, F.; Yang, L. Robust nucleus/cell detection and segmentation in digital pathology and microscopy images: A comprehensive review. IEEE Rev. Biomed. Eng. 2016, 9, 234-263. [CrossRef] [PubMed]

7. Mignotte, M. Segmentation by fusion of histogram-based $k$-means clusters in different color spaces. IEEE Trans. Image Process. 2008, 17, 780-787. [CrossRef] [PubMed]

8. Hathaway, R.J.; Bezdek, J.C. Local convergence of the fuzzy c-means algorithms. Pattern Recognit. 1986, 19, 477-480. [CrossRef]

9. Krishna, K.; Murty, M.N. Genetic K-means algorithm. IEEE Trans. Syst. Man Cybern. Part B (Cybern.) 1999, 29, 433-439. [CrossRef] [PubMed]

10. Rana, S.; Jasola, S.; Kumar, R. A review on particle swarm optimization algorithms and their applications to data clustering. Artif. Intell. Rev. 2011, 35, 211-222. [CrossRef]

11. Chowdhury, A.; Bose, S.; Das, S. Automatic clustering based on invasive weed optimization algorithm. In International Conference on Swarm, Evolutionary, and Memetic Computing; Springer: Berlin/Heidelberg, Germany, 2011; pp. 105-112.

12. Rao, R.V.; Savsani, V.J.; Vakharia, D.P. Teaching-learning-based optimization: An optimization method for continuous non-linear large scale problems. Inf. Sci. 2012, 183, 1-5. [CrossRef]

13. Patra, B.; Chen, Y.H.; Peng, C.C.; Lin, S.C.; Lee, C.H.; Tung, Y.C. A microfluidic device for Funiform-sized cell spheroids formation, culture, harvesting and flow cytometry analysis. Biomicrofluidics 2013, 7. [CrossRef]

14. Abràmoff, M.D.; Magalhães, P.J.; Ram, S.J. Image processing with ImageJ. Biophotonics Int. 2004, 11, 36-42.

15. Alkasalias, T.; Moyano-Galceran, L.; Arsenian-Henriksson, M.; Lehti, K. Fibroblasts in the tumor microenvironment: Shield or spear? Int. J. Mol. Sci. 2018, 19, 1532. [CrossRef]

16. Fang, Y.; Eglen, R.M. Three-Dimensional Cell Cultures in Drug Discovery and Development. SLAS Discov. 2017, 22, 456-472. [CrossRef]

17. Lv, D.; Hu, Z.; Lu, L.; Lu, H.; Xu, X. Three-dimensional cell culture: A powerful tool in tumor research and drug discovery. Oncol. Lett. 2017, 14, 6999-7010. [CrossRef] [PubMed]

18. Kim, S.H.; Lee, G.H.; Park, J.Y. Microwell fabrication methods and applications for cellular studies. Biomed. Eng. Lett. 2013, 3, 131-137. [CrossRef]

19. Schwarz, M.W.; Cowan, W.B.; Beatty, J.C. An experimental comparison of RGB, YIQ, LAB, HSV, and opponent color models. ACM Trans. Graph. (TOG) 1987, 6, 123-158. [CrossRef]

20. Vala, H.J.; Baxi, A. A review on Otsu image segmentation algorithm. Int. J. Adv. Res. Comput. Eng. Technol. (IJARCET) 2013, 2, 387-389.

21. Naik, A.; Satapathy, S.C.; Parvathi, K. Improvement of initial cluster center of c-means using teaching learning based optimization. Procedia Technol. 2012, 6, 428-435. [CrossRef]

22. Grau, V.; Mewes, A.U.; Alcaniz, M.; Kikinis, R.; Warfield, S.K. Improved watershed transform for medical image segmentation using prior information. IEEE Trans. Med. Imaging 2004, 23, 447-458. [CrossRef] [PubMed]

23. Zanoni, M.; Piccinini, F.; Arienti, C.; Zamagni, A.; Santi, S.; Polico, R.; Bevilacqua, A.; Tesei, A. 3D tumor spheroid models for in vitro therapeutic screening: A systematic approach to enhance the biological relevance of data obtained. Sci. Rep. 2016, 6, 1. [CrossRef] [PubMed]

\section{Short Biography of Authors}

Mukta Sharma received a bachelor's degree in computer science from Uttar Pradesh Technical University, India. She completed her master's degree from GLA University, India. She is currently pursuing her doctorate from the Department of Information Technology at Atal Bihari Vajpayee Indian Institute of Information Technology and Management, Gwalior, India. Under her Ph.D. studies, Mukta Sharma is actively working on the segmentation and classification of histopathological images of cells in biomedical imaging. She has published papers in several conferences and refereed journals such as MBEC, Journal of Supercomputing, Springer. Her areas of interest are image processing, computer vision, machine learning for biomedical applications, cell morphological analysis, deep learning, and medical imaging. She has a currently active IEEE membership. Contact her at mukta.24sharma@gmail.com. 
Venkanagouda S. Goudar is presently a Ph.D. student under the guidance of Prof. Fan-Gang Tseng in engineering and system sciences, National Tsing Hua University, Hsinchu, Taiwan. He completed his M.Sc. degree in Microbiology from Karnataka University, Dharwad, India in 2009. He further worked as a project assistant at the Indian Institute of Science, Bangalore, India for 4 years. He later worked as a research trainee for 7 months at the Institute of Photonic Sciences (ICFO), Barcelona, Spain. During this period, he was involved in different research areas such as nanotechnology, biosensors, lab-on-a-chip, and antibacterial and antiviral surface modifications on transparent surfaces. Currently, he is working on rare cell 3D culturing and drug delivery. Overall, during his research, he was able to produce eight peer-reviewed journal publications (Sensors and Actuators, AMI, IEEE sensors, etc.) and seven conference abstracts, and to file three patents (Indian, Taiwan, and the U.S.) in the field of biosensors and drug delivery. Contact him at venkatesh.gdr23@gmail.com.

Manohar Prasad Koduri is a dual Ph.D. student of the National Tsing Hua University, Taiwan and the University of Liverpool, UK. Manohar completed his masters in integrated circuit technologies at the University of Hyderabad, India, and finished his bachelor's degree in electronics and communication engineering at Gayatri Vidya Parishad College of Engineering, Visakhapatnam, India. Manohar is currently working on developing nanosensors for 3D tissue engineering applications. His research interests include biosensors, drug delivery, electromagnetic theory, image processing, and tissue engineering. Contact him at manimanohar92@gmail.com.

Prof. Tseng Fan-Gang is presently the vice president for R\&D, NTHU, leading a multidisciplinary team at national Tsing Hua University, Hsinchu, Taiwan. For the past 20 years, his research interest included BioMEMS, nano/microfluidics, biosensors, Microfuel cells, and hydrogen storage. His recent research in BioMEMS involves cancer marker diagnosis and drug delivery, single-cell diagnosis, CTC diagnosis, the study of single-cell protein and cell dynamics, and microbubble generation and the study of its applications in tissue engineering and cancer research. He was elected as a fellow of ASME in 2014. He has received 69 patents, written 9 book chapters, published more than 220 SCI/EI journal papers with an $\mathrm{H}$ index 41, and has more than 6774 citations and 360 conference technical papers in the related fields. Prof. Tseng co-organized or cochaired many conferences, including Micro TAS, ISMM, IEEE MEMS, IEEE NEMS, IEEE Transducers, IEEE Nano, and IEEE Nanomed. Contact him at fangangtseng@gmail.com.

Prof. Mahua Bhattacharya is currently working as a Full Professor of the ABV Indian Institute of Information Technology and Management, Gwalior. The research area of Prof. Bhattacharya is related to biomedical-image processing, the classification of tumor or cancer growth in the human brain using multimodality medical imaging, cell-image analysis under various types of environmental exposure, and the development of AI techniques for digital and smart farming. She is executing various Government of India-funded projects in collaboration with reputed institutes. She is the President of the International Neural Network Society, India Chapter. Prof. Bhattacharya has published more than 150 papers in refereed journals and international flagship conferences as book chapters. She is the reviewer of IEEE EMBC, Elsevier, Springer, and Taylor and Francis journals. She is an organizing committee member of international IEEE conferences in India and abroad. She has delivered expert lectures in different national and international academic forums. She is an editorial board member of Neural Computing and Applications, Springer. She was Indian liaison for IJCNN'19 in Budapest, Hungary. Contact her at mahuabhatta@gmail.com. 